\title{
Evaluación de la función endotelial y la función diastólica en pacientes con hiperparatiroidismo primario antes y después de la paratiroidectomía
}

\author{
Evaluation of endothelial function and diastolic function in patients with primary \\ hyperparathyroidism before and after parathyroidectomy
}

\author{
Roser M. Magriñá-Mercado', Claudia Ramírez-Rentería², Eduardo Almeida-Gutiérrez", \\ Victoria Mendoza-Zubieta ${ }^{1}$ y Aldo Ferreira-Hermosillo2* \\ ${ }^{1}$ Servicio de Endocrinología; ${ }^{2}$ Unidad de Investigación en Endocrinología Experimental. Hospital de Especialidades Centro Médico Nacional Siglo \\ XXI; ${ }^{3}$ Coordinación de Investigación en Salud, Instituto Mexicano del Seguro Social. Ciudad de México, México
}

\begin{abstract}
Resumen
Introducción: El hiperparatiroidismo primario (HPTP) es la principal causa de hipercalcemia ambulatoria y se ha asociado con un riesgo cardiovascular elevado. La vasodilatación mediada por flujo (VMF) es un método no invasivo que evalúa la función endotelial. Objetivo: Comparar la disfunción endotelial mediante VMF y la disfunción diastólica en pacientes con HPTP antes y después de la paratiroidectomía. Método: Mediante un estudio cuasiexperimental (antes-después) se evaluaron la función diastólica y la VMF antes y 6 meses después de realizar una paratiroidectomía. Resultados: Se incluyeron 15 pacientes con HPTP: 12 mujeres (80\%) y 3 hombres (20\%); el 73\% presentaron litiasis, el 27\% osteoporosis y el 53\% síndrome metabólico; el $73 \%$ tenían disfunción diastólica antes de la cirugía y el $60 \%$ después de la cirugía $(p=0.09)$. Los diámetros braquiales post-isquemia mejoraron de $41 \mathrm{~mm}$ antes de la cirugía a $46 \mathrm{~mm}$ tras la cirugía $(p=0.020)$. Así mismo, los diámetros braquiales preisquemia vs. post-isquemia después de la cirugía mejoraron de 41 a $46 \mathrm{~mm}(p=0.005)$. Antes de la cirugía, el cambio en la delta del diámetro braquial preisquemia y post-isquemia fue de $1 \mathrm{~mm}$, y subió a $4 \mathrm{~mm}$ después de la cirugía $(p=0.03)$. Conclusiones: Existe una menor disfunción endotelial medida por VMF en pacientes posoperados por HPTP a los 6 meses de la cirugía. El estudio ecocardiográfico puede ser de utilidad en la evaluación preoperatoria de los pacientes con HPTP asintomáticos.
\end{abstract}

PALABRAS CLAVE: Vasodilatación. Endotelio vascular. Hiperparatiroidismo primario. Paratiroidectomía.

\begin{abstract}
Introduction: Primary hyperparathyroidism (PHPT) is the leading cause of outpatient hypercalcemia associated with increased cardiovascular risk. The flow-mediated vasodilation (FMV) has been proposed as a non-invasive method for assessing endothelial function. Objective: To compare the endothelial dysfunction measured by FMV and diastolic dysfunction in patients with PHPT before and after parathyroidectomy. Method: We performed a quasi-experimental (before-after) study to evaluate diastolic function and FMV in patients with PHPT before and six months after parathyroidectomy. Results: Fifteen patients completed study: 12 women and 3 men; $73 \%$ presented lithiasis, 27\% osteoporosis and 53\% metabolic syndrome; $73 \%$ presented diastolic dysfunction before the surgery and $60 \%$ after the surgery $(p=0.09)$. Post-isquemia brachial diameter improved from $41 \mathrm{~mm}$ (before surgery) to $46 \mathrm{~mm}$ (after surgery; $p=0.020$ ). After surgery, we also observed an increase in the brachial dia-
\end{abstract}

\author{
Correspondencia: \\ *Aldo Ferreira-Hermosillo \\ Cuauhtémoc, 330 \\ Col. Doctores, Del. Cuauhtémoc \\ C.P. 06720, Ciudad de México, México \\ E-mail: aldo.nagisa@gmail.com
}

Fecha de recepción: 09-06-2018

Fecha de aceptación: 20-09-2018

DOI: $10.24875 / C I R U .18000520$
Cir Cir. 2019;87:196-204

Contents available at PubMed www.cirugiaycirujanos.com 
meter pre vs. post-ischemia from 41 to $46 \mathrm{~mm}(p=0.005)$. Before surgery, the change in the delta of brachial diameter pre and post-ischemia was $1 \mathrm{~mm}$ and up to $4 \mathrm{~mm}$ after surgery $(p=0.03)$. Conclusions: There is a minor endothelial dysfunction measured by FMV in patients who underwent surgery for PHPT at 6 months after surgery, as well as a trend towards improvement in diastolic dysfunction. Echocardiography can be useful in the preoperative evaluation in patients with asymptomatic PHPT.

KEY WORDS: Vasodilation. Vascular endothelium. Primary hyperparathyroidism. Parathyroidectomy.

\section{Introducción}

Se estima que, en la actualidad, el hiperparatiroidismo primario (HPTP) es el tercer trastorno endocrinológico más frecuentemente diagnosticado, con una incidencia de 21 por 1000 habitantes ${ }^{1}$. Es la causa principal de hipercalcemia en pacientes ambulatorios, y su prevalencia se calcula en 1 a 4 por cada 1000 personas en la población general ${ }^{2,3}$. Se ha relacionado con un aumento del riesgo de mortalidad cardiovascular-6 y a menudo se asocia con hipertensión, dislipidemia y resistencia a la insulina, que constituyen factores del síndrome metabólico ${ }^{7-9}$. De hecho, en un estudio realizado en población mexicana, el 59\% de los pacientes con HPTP fueron diagnosticados de síndrome metabólico utilizando los criterios de consenso $0^{10}$; sin embargo, la relación entre las alteraciones bioquímicas en el HPTP y el riesgo de complicaciones cardiovasculares no ha sido dilucidada por completo.

La mayoría de los factores de riesgo cardiovascular asociados al HPTP se han evaluado en estudios observacionales ${ }^{11}$. Procopio, et al. ${ }^{12}$ evaluaron el riesgo cardiovascular en los pacientes con HPTP mediante la escala de Framingham, dividiendo a la población en asintomáticos (con alto y bajo riesgo para daño de órgano diana) y sintomáticos (con enfermedad ósea o renal, o crisis hipercalcémica) y comparando con pacientes controles. En este estudio, el $52 \%$ de los pacientes con HPTP tuvieron un riesgo cardiovascular intermedio y el $21 \%$ presentaban síndrome metabólico, mientras que el $65 \%$ de los pacientes con HPTP sintomáticos y el $58 \%$ de los asintomáticos presentaron un riesgo cardiovascular elevado.

Actualmente, la decisión de realizar una cirugía en los pacientes con HPTP subclínico se determina de acuerdo con los criterios del Cuarto Consenso Internacional ${ }^{13}$. Este consenso incluyó las opiniones de un grupo de expertos de la Endocrine Society que se reunieron en Italia en el año 2013, y fue elaborado considerando la evidencia científica proveniente de cuatro artículos principales sobre HPTP subclínico.
A pesar de su utilidad como guía clínica, dicho consenso incluye poca información respecto a la decisión orientada a la mejoría del efecto cardiovascular, sobre todo en pacientes con HPTP leve. Walker, et al. ${ }^{14}$ observaron una mejoría en la distensibilidad vascular carotídea en 44 pacientes a los 2 años de la paratiroidectomía. En estos pacientes también se evaluó la mejoría de la disfunción endotelial mediante la vasodilatación mediada por flujo (VMF). En un trabajo llevado a cabo por Carrelli, et al..$^{15}$ se estudió a 46 pacientes con HPTP leve antes y a los 6 y 12 meses de la paratiroidectomía, y se observó que 15 pacientes con disfunción endotelial presentaron una mejoría del $350 \%$. En otros estudios se ha encontrado un decremento del índice de masa del ventrículo izquierdo ${ }^{16} 0$ una regresión de la hipertrofia del tabique interventricular y de la pared posterior ${ }^{17}$ después de la cirugía.

La evaluación del riesgo cardiovascular en el HPTP se ha realizado por métodos como el grosor carotídeo íntima-media ${ }^{11}$, mediante la determinación del propéptido natriurético cerebral $\mathrm{N}$-termina ${ }^{18}$ y mediante la respuesta inducida por nitroglicerina ${ }^{19}$; sin embargo, pocos estudios han usado la VMF. El objetivo de este estudio fue evaluar la VMF y la función diastólica en pacientes con HPTP antes y 6 meses después de la paratiroidectomía.

\section{Método}

\section{Procedimientos generales}

Se realizó un estudio cuasiexperimental, prospectivo y analítico de 15 pacientes con diagnóstico de HPTP de la Clínica de Calcio del Servicio de Endocrinología del Hospital de Especialidades del Centro Médico Nacional Siglo XXI. Se incluyeron pacientes mayores de 16 años, con HPTP, que fueran candidatos a paratiroidectomía (de acuerdo con los criterios del Cuarto Consenso Internacional $\left.{ }^{13}\right)$. Se excluyeron los pacientes con cirugía paratiroidea previa 0 antecedente de cardiopatía. Los pacientes fueron invitados a participar en el estudio y, después de explicárseles el objetivo de este, firmaron la carta de 
consentimiento informado de acuerdo con los lineamientos del Comité Local de Investigación en Salud (Número de Registro F-2014-3601-218). Se les realizó historia clínica, exploración física completa con énfasis en antropometría, y toma de muestras para pruebas de laboratorio.

\section{Evaluación antropométrica}

Se registraron el peso (kilos), la talla (metros) y el perímetro de la cintura $(\mathrm{cm})$. Un investigador único (AFH), utilizando la misma báscula y el mismo estadímetro, realizó todas las medidas antropométricas. El perímetro de la cintura se midió en el punto medio entre la última costilla y la espina ilíaca anterosuperior. El índice de masa corporal (IMC) se calculó dividiendo el peso entre la estatura al cuadrado. Se calculó, además, el índice cintura/estatura. La presión arterial se determinó en el brazo izquierdo, después de 10 minutos de descanso, en posición de sentado, en ayuno, sin consumo previo de café ni tabaco.

\section{Criterios diagnósticos de síndrome metabólico}

Se definió el síndrome metabólico utilizando los criterios del consenso de la American Heart Association/National Heart Lung and Blood Institute y la International Diabetes Federation. Se tomaron tres o más de los siguientes criterios: triglicéridos séricos $>150 \mathrm{mg} / \mathrm{dl}(1.7 \mathrm{mmol} / \mathrm{l})$, colesterol ligado a lipoproteínas de alta densidad HDL (C-HDL) $<40 \mathrm{mg} / \mathrm{dl}$ $(1.03 \mathrm{mmol} / \mathrm{l})$ en hombres y $<50 \mathrm{mg} / \mathrm{dl}(1.29 \mathrm{mmol} / \mathrm{l})$ en mujeres 0 en tratamiento para dislipidemias, presión arterial $>130 / 85 \mathrm{mmHg}$ o en tratamiento con antihipertensivos, perímetro de la cintura $>90 \mathrm{~cm}$ en hombres $0>80 \mathrm{~cm}$ en mujeres, 0 alteraciones del metabolismo de la glucosa (prediabetes o diabetes, de acuerdo con los criterios de la American Diabetes Association $)^{20}$.

\section{Evaluación bioquímica}

Se solicitaron estudios de laboratorio después de un periodo de 8 horas de ayuno. Se determinaron la glucosa, el colesterol total, el C-HDL y los triglicéridos mediante técnica de fotocolorimetría, utilizando kits comerciales. Para obtener la concentración de C-HDL se utilizó la prueba enzimática de precipitación con polietilenglicol y dextrano sulfato, y se analizó con la misma técnica fotocolorimétrica que el colesterol. La hemoglobina glucosilada se determinó mediante inmunoanálisis. La concentración de colesterol ligado a lipoproteínas de baja densidad se calculó mediante la fórmula de Friedewald²1.

\section{Evaluación cardiovascular}

La evaluación cardiovascular estuvo a cargo de un cardiólogo ecocardiografista. Se realizó ecocardiograma 2D para medir los índices de función sistólica y diastólica del ventrículo izquierdo (diámetro anteroposterior, diámetro diastólico, diámetro sistólico, fracción de eyección), y mediante Doppler pulsado se midió el flujo diastólico transmitral para determinar las velocidades máximas de onda temprana y tardía (onda $\mathrm{E}$ y onda A) y su relación (E/A), el tiempo de desaceleración de la onda $\mathrm{E}$ y el tiempo de relajación isovolumétrica. Además, se determinó el diámetro basal (preisquemia) de la arteria braquial (previa fijación del brazo dominante y detección de la arteria en la fosa antecubital) y tras la isquemia (posterior a inflar el manguito del esfigmomanómetro $20-50 \mathrm{mmHg}$ por arriba de la presión arterial sistólica y esperar 5 minutos, desinflar el manguito, volver a esperar 1 minuto, y finalmente registrar el diámetro de la luz arterial), considerando como normal un aumento del diámetro tras la isquemia mayor del $10 \%{ }^{15,22}$. Estas mediciones fueron repetidas a los 6 meses de la paratiroidectomía.

\section{Análisis estadístico}

Se calculó el tamaño de la muestra utilizando la fórmula de diferencia de medias, con los estimadores referidos en el artículo de Carrelli, et al. ${ }^{15}$; considerando que en su grupo de pacientes con HPTP y disfunción endotelial se presentó un incremento en la VMF del $0.81 \pm 0.19 \%$ al $3.18 \pm 0.79 \%$ a los 6 meses, se obtuvo un cálculo de 15 pacientes.

Las variables cuantitativas se describieron utilizando medidas de tendencia central y de dispersión, de acuerdo con la distribución de los datos. Las variables cualitativas se refirieron utilizando frecuencias absolutas y relativas. Para establecer la normalidad en la distribución de las variables cuantitativas se utilizó la prueba de Shapiro-Wilk. Para las asociaciones entre las variables cualitativas relacionadas se utilizó la prueba de McNemar, y para las variables cuantitativas se utilizó la prueba de t pareada o la prueba de rangos con signo de Wilcoxon, de acuerdo con su distribución. Se consideró un valor de $p<0.05$ para establecer la significancia estadística. 


\section{Resultados}

Se incluyeron 15 pacientes con estudios antes y después de la cirugía; $12(80 \%)$ de ellos eran mujeres y $3(20 \%)$ eran hombres; la mediana de edad fue de 54 años (rango intercuartílicos [RI]: $47-70$ ) y el $26.7 \%$ eran menores de 50 años. La mediana del IMC fue de $26.6 \mathrm{~kg} / \mathrm{m}^{2}$ (Rl: $23.3-28.3$ ); solo el $40 \%$ de los pacientes tenía peso normal, el $53 \%$ presentaban sobrepeso y el $7 \%$ tenían obesidad de grado I, es decir, con IMC de $25.1-29.9 \mathrm{~kg} / \mathrm{m}^{2}$ y $30-34.9 \mathrm{~kg} / \mathrm{m}^{2}$, respectivamente.

Dentro de las manifestaciones del HPTP, el $73 \%$ tenían litiasis y el $27 \%$ presentaban osteoporosis. Además, el $20 \%$ se presentaron con una depuración de creatinina $<60 \mathrm{ml} / \mathrm{min}$, y $1(7 \%)$ paciente era portador de trasplante renal de donador vivo relacionado (esposa). El $60 \%$ tenían unas cifras de calcio por encima de $11.2 \mathrm{mg} / \mathrm{dl}$ y el $40 \%$ presentaban hipercalcemia leve (10.7-11.2 mg/dl). La mediana de la evolución de los síntomas del HPTP fue de 4 años (RI: 1.59 años).

Entre los factores de riesgo cardiovascular se encontró que el $73 \%$ eran hipertensos, el $20 \%$ tenían diagnóstico de diabetes y el $53 \%$ de prediabetes, el $13 \%$ tenían dislipidemia y el $27 \%$ antecedente de tabaquismo. Solo una paciente presentó una cintura $<80 \mathrm{~cm}$ y todos los demás tuvieron una cintura mayor que lo recomendado por sexo. El índice cintura/estatura en los hombres fue de 61.5 (55.8-61.5) y en las mujeres de 59.4 (53.4-63.8). Todos los hombres tuvieron un índice cintura/estatura $>0.52$, tomando como referencia los criterios de Ferreira-Hermosillo, et al. ${ }^{23}$ para detección de síndrome metabólico. El $92 \%$ de las mujeres también tuvieron este índice elevado. Finalmente, de acuerdo con los criterios de consenso, el 53\% de los pacientes presentaron síndrome metabólico.

Respecto a las variables de síndrome metabólico, se observó una mejoría en las concentraciones de glucosa, de ácido úrico y de colesterol total después de la cirugía; sin embargo, esto no fue estadísticamente significativo $(p>0.05)$. No se observaron diferencias en la prevalencia de síndrome metabólico entre antes y después de la cirugía.

En la tabla 1 se presentan los resultados de las variables del metabolismo del calcio y la vitamina $D$, así como otras variables bioquímicas y del ecocardiograma.

Con relación a las variables ecocardiográficas al momento del diagnóstico, se encontró que los diámetros de la aurícula derecha, el diámetro basal del ventrículo derecho, la raíz aórtica, la presión sistólica de la arteria pulmonar, el diámetro diastólico del ventrículo izquierdo, el diámetro sistólico del ventrículo izquierdo, la pared posterior del ventrículo izquierdo, la fracción de eyección del ventrículo izquierdo y el desplazamiento sistólico del anillo tricuspídeo se encontraban dentro de los parámetros normales (Tabla 1) de acuerdo con las recomendaciones para la cuantificación de las cámaras cardiacas por ecocardiografía de la American Society of Echocardiography y la Asociación Europea de Imagen Cardiovascular ${ }^{24}$, con excepción de dos pacientes que tenían dilatación auricular izquierda y seis pacientes con tabique y pared posterior $>10 \mathrm{~mm}$ que indicaban hipertrofia concéntrica del ventrículo izquierdo; estos pacientes tenían diagnóstico previo de hipertensión (cardiopatía hipertensiva).

En cuanto a las variables ecocardiográficas evaluadas antes y después de la cirugía, no se encontraron diferencias en el diámetro anteroposterior de la aurícula izquierda, el tiempo de relajación isovolumétrica del ventrículo izquierdo, las ondas $E$ y $A$ del ventrículo izquierdo, la ratio E/A ni el tiempo de desaceleración del ventrículo izquierdo (Tabla 1). Se determinó la presencia de disfunción diastólica en el $73 \%$ de los pacientes antes de la cirugía, y posterior a esta en el $60 \%(p=0.09)$.

La mediana del diámetro de la arteria braquial preisquemia antes de la cirugía fue de $38 \mathrm{~mm}$ (RIC: $36-46 \mathrm{~mm}$ ), y posterior a la cirugía fue de $41 \mathrm{~mm}$ (RIC: 37-48 mm; $p=0.258$ ); el diámetro post-isquemia antes de la cirugía fue de $41 \mathrm{~mm}$ (RIC: $32-46 \mathrm{~mm}$ ), y posterior a la cirugía fue de $46 \mathrm{~mm}$ (RIC: $40-51 \mathrm{~mm} ; \mathrm{p}=0.02$ ) (Fig. 1). Después de 6 meses de haberse sometido a paratiroidectomía se observó una mejoría de la vasodilatación arterial braquial (diámetros braquiales preisquemia vs. post-isquemia después de la cirugía de 41 [Rl: $37-48 \mathrm{~mm}$ ] a $46 \mathrm{~mm}$ [Rl: $40-51 \mathrm{~mm}] ; p=0.005$ ).

En la figura $2 \mathrm{~A}$ se observa que antes de la paratiroidectomía solo tres pacientes tuvieron disminución del diámetro post-isquemia y un paciente permaneció sin cambios, mientras que después de la paratiroidectomía 14 de 15 pacientes tuvieron aumento del diámetro braquial post-isquemia y solo un paciente permaneció sin cambios (Fig. 2 B).

Un resultado muy relevante fue la delta de cambio en el diámetro braquial entre antes y después de la isquemia: previo a la cirugía, los pacientes tuvieron una delta de solo $1 \mathrm{~mm}$ (RI: $-2-4 \mathrm{~mm})$, y después de la cirugía la delta subió a $4 \mathrm{~mm}(\mathrm{RI}: 1-7 \mathrm{~mm} ; \mathrm{p}=0.03)$ (Fig. 3). 
Tabla 1. Comparación entre los resultados bioquímicos y la evaluación vascular ecocardiográfica de los pacientes en el momento del diagnóstico (prequirúrgico) y posterior (post-quirúrgico) a la paratiroidectomía $(n=15)$

\begin{tabular}{|c|c|c|c|}
\hline Parámetro & Prequirúrgico & Post-quirúrgico & $\mathrm{p}$ \\
\hline Calcio, mg/dl & $11.4(11-12)$ & $9.1(8.34-9.6)$ & 0.001 \\
\hline Fósforo, mg/dl & $2.3(2.0-2.7)$ & $3.4(3.0-3.6)$ & 0.002 \\
\hline PTH intacta, pg/ml & $162(126.1-318.3)$ & $20.4(17.7-28.1)$ & 0.001 \\
\hline Vitamina $D, n g / d l$ & $15.8(8.8-18.0)$ & $17.8(12.9-18.8)$ & 0.173 \\
\hline Creatinina, mg/dl & $0.81(0.75-1.06)$ & ND & \\
\hline $\begin{array}{l}\text { Depuración de creatinina, } \\
\mathrm{ml} / \mathrm{min}\end{array}$ & $99.0(72.0-113.7)$ & ND & \\
\hline Calcio urinario, mg/24 h & $252.0(150.0-400.0)$ & ND & \\
\hline Glucosa, mg/dl & $103(94-116)$ & $95(91-128)$ & 0.691 \\
\hline Ácido úrico, mg/dl & $5.9(4.8-8.1)$ & $5.3(4.3-6.6)$ & 0.109 \\
\hline Colesterol total, mg/dl & $191(146-209)$ & $184(161-204)$ & 0.244 \\
\hline $\mathrm{C}-\mathrm{HDL}, \mathrm{mg} / \mathrm{dl}$ & $49(35-50)$ & $50(35-55)$ & 0.720 \\
\hline C-LDL, mg/dl & $112(66-130)$ & $99(85-111)$ & 0.720 \\
\hline Triglicéridos, mg/dl & $157(123-196)$ & $186(122-249)$ & 0.842 \\
\hline Diámetro AP de la Al, mm & $32(31-33)$ & $33(32-36)$ & 0.123 \\
\hline AD (diámetro mayor), mm & $34(31.5-36.5)$ & ND & \\
\hline $\mathrm{VD}, \mathrm{mm}$ & $26.0(24.8-29)$ & ND & \\
\hline Raíz aórtica, mm & $29(25.5-32.5)$ & ND & \\
\hline PSAP, mmHg & $29(25-31)$ & ND & \\
\hline DDVI, mm & $43(41-48)$ & ND & \\
\hline DSVI, mm & $28(24-29)$ & ND & \\
\hline PPVI, mm & $10(9-13)$ & ND & \\
\hline FEVI, \% & $64(62-65)$ & ND & \\
\hline TAPSE, mm & $23(20-24)$ & ND & \\
\hline TRIVI, ms & $95(70-110)$ & $105(80-125)$ & 0.162 \\
\hline Onda $\mathrm{E}, \mathrm{mm} / \mathrm{s}$ & $73(59-77)$ & $66(59-74)$ & 0.378 \\
\hline Onda A, mm/s & $79(57-94)$ & $75(63-87)$ & 0.593 \\
\hline Relación E/A, mm/s & 0.92 & 0.83 & 0.100 \\
\hline $\mathrm{TD}, \mathrm{ms}$ & $165(150-205)$ & $185(165-225)$ & 0.190 \\
\hline
\end{tabular}

Los resultados se presentan como mediana (rango intercuartilico).

AD: aurícula derecha; Al: aurícula izquierda; AP: anteroposterior; C-HDL: colesterol asociado a lipoproteínas de alta densidad; C-LDL: colesterol asociado a lipoproteínas de baja densidad; DDVI: diámetro diastólico del ventrículo izquierdo; DSVI: diámetro sistólico del ventrículo izquierdo; FEVI: fracción de eyección del ventrículo izquierdo; ND: no determinado;

PPVI: pared posterior del ventrículo izquierdo; PSAP: presión sistólica de la arteria pulmonar; PTH: hormona paratiroidea; TAPSE: desplazamiento sistólico del anillo tricuspídeo; TD:

tiempo de desaceleración; TRIVI: tiempo de relajación isovolumétrica del ventrículo izquierdo; VD: ventrículo derecho.

\section{Discusión}

Se ha considerado que el HPTP es un factor que incrementa la morbilidad cardiovascular; sin embargo, aún existe controversia en cuanto al beneficio de una cirugía en aquellos pacientes con HPTP leve asintomáticos.
A pesar de las asociaciones encontradas con la hipercalcemia, la vitamina $D$ y los componentes del síndrome metabólico, aún no se ha determinado el mecanismo fisiopatológico por el cual el corazón sufre daño en los pacientes con HPTP ${ }^{10}$. Se sugiere que el endotelio es un órgano diana para la 


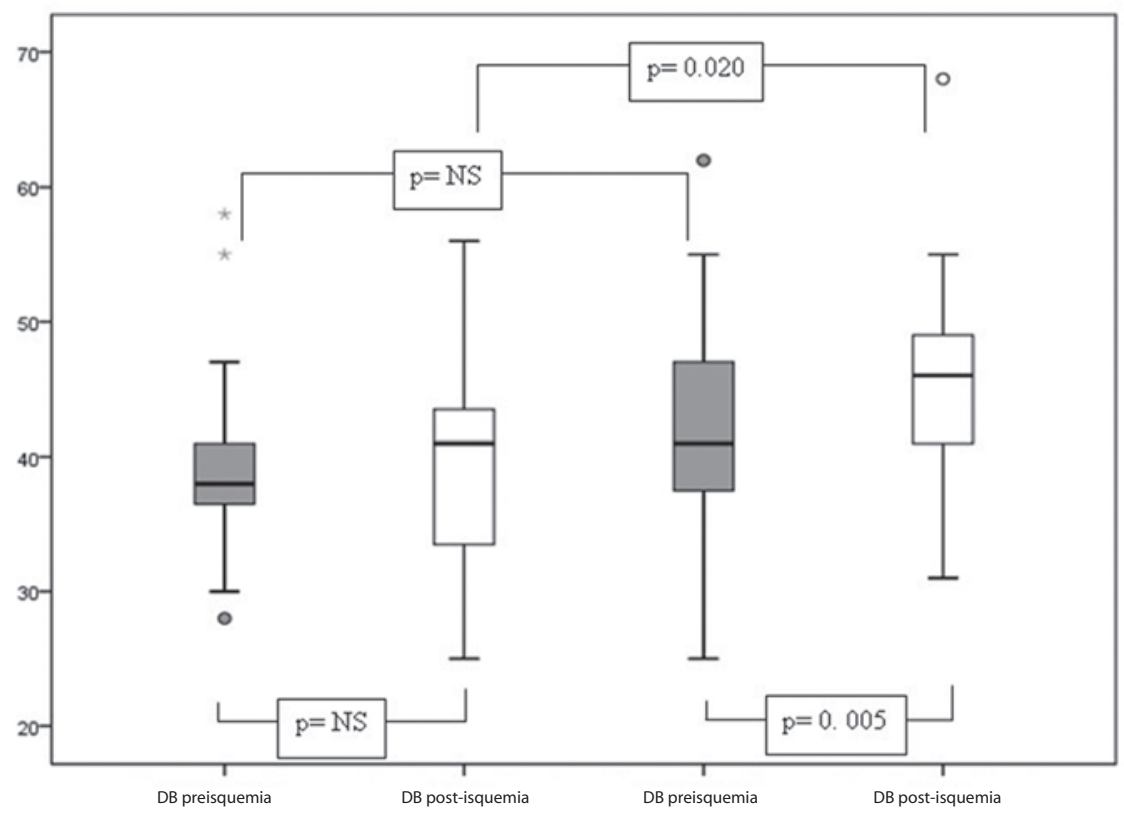

Figura 1. Comparación del cambio de diámetro de la arteria braquial (DB) durante la prueba de vasodilatación mediada por flujo en pacientes con hiperparatiroidismo primario antes y después de la cirugía. Se observa que los DB preisquemia no presentaron diferencia significativa (NS), pero los diámetros post-isquemia aumentaron después de la paratiroidectomía. Sí se encuentra diferencia significativa entre el diámetro basal preisquemia y post-isquemia después de la paratiroidectomía.

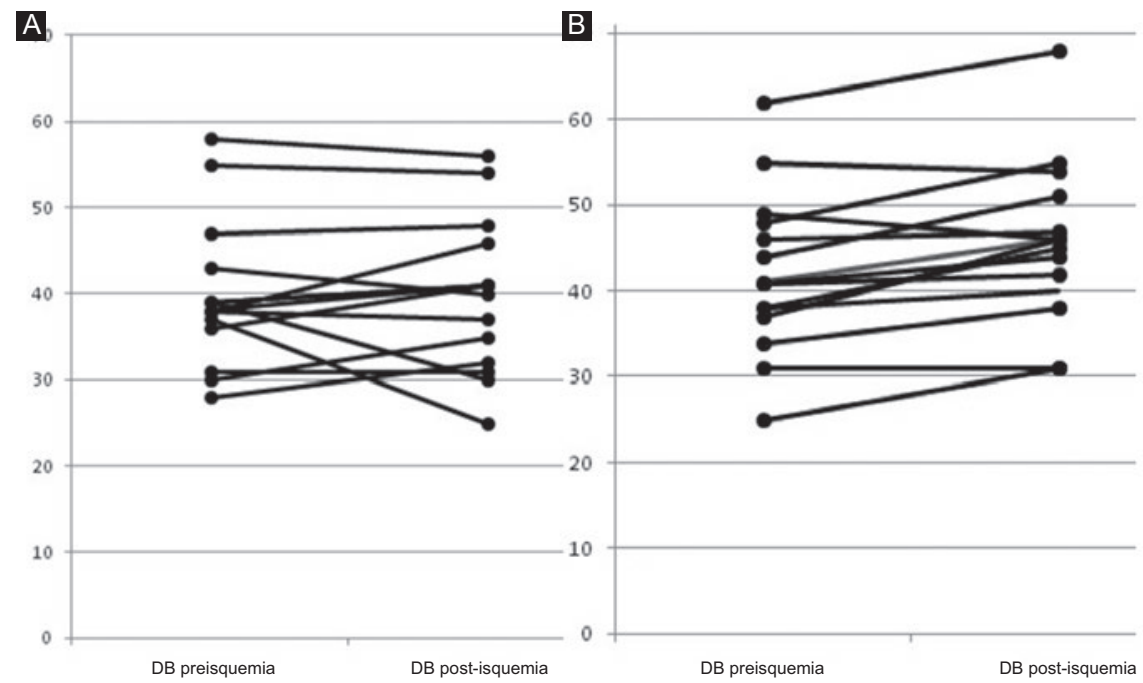

Figura 2. Diámetros braquiales (DB) durante la prueba de la vasodilatación mediada por flujo en pacientes con hiperparatiroidismo primario antes $(A)$ y después $(B)$ de la paratiroidectomía. Se muestra que antes de la cirugía solo tres pacientes tuvieron disminución del $D B$ posterior a la isquemia y un paciente permaneció sin cambios, mientras que después de la paratiroidectomía 14 pacientes aumentaron el diámetro braquial y solo uno permaneció sin cambios.

hormona paratiroidea, la cual puede estimular la proliferación de músculo liso y de cardiomiocitos, además de contribuir a la aterogénesis por influencia directa sobre la calcificación vascular y la remodelación ${ }^{25,26}$. Otra teoría ha propuesto que el daño es secundario a la deficiencia de vitamina $D$ debido a la activación del eje renina-angiotensina-aldosterona y la secreción de moléculas proinflamatorias $^{12}$.
Una de las dificultades para evaluar el riesgo cardiovascular en estos pacientes radica en la elevada prevalencia de factores de riesgo como la dislipidemia y la hipertensión en la población mexicana. En general, los pacientes mayores de 40 años en nuestro país tienen más tiempo de evolución de estas enfermedades, mientras que el tiempo de exposición a la hipercalcemia y la elevación de la hormona paratiroidea en los pacientes con HPTP es relativamente corto. 


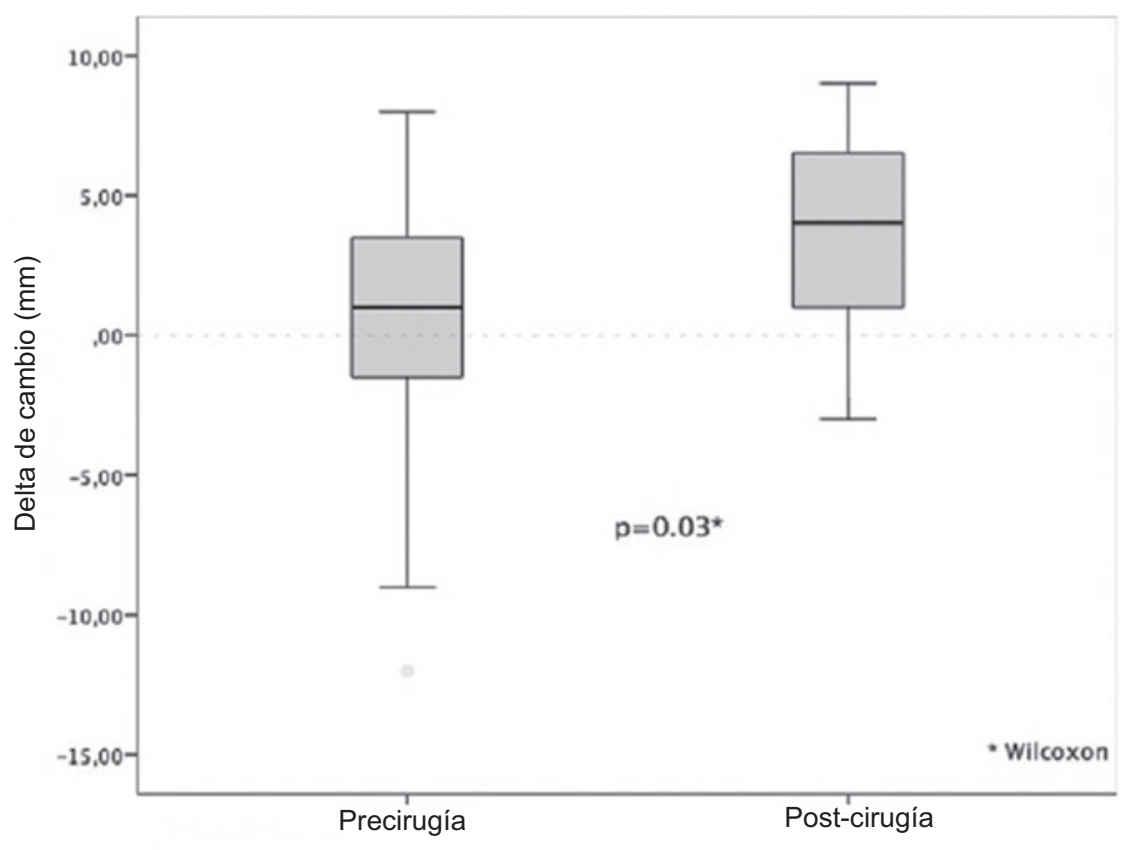

Figura 3. Comparación de la delta de cambio del diámetro de la arteria braquial durante la prueba de vasodilatación mediada por flujo en pacientes con hiperparatiroidismo primario antes y después de la paratiroidectomía. La delta de cambio antes de la cirugía fue de $1 \mathrm{~mm}$ (rango intercuartílico: -2-4 mm) y después de la cirugía aumentó a $4 \mathrm{~mm}$ (rango intercuartílico: $1-7 \mathrm{~mm} ; p=0.03$ ).

La evaluación del riesgo cardiovascular se ha realizado por varios métodos, entre ellos la VMF. Esta técnica evalúa la función endotelial dependiente del óxido nítrico y se asocia con la enfermedad aterosclerosa coronaria ${ }^{15,22}$. En la población de pacientes estudiados tiene tres ventajas: 1) la disfunción endotelial es uno de los primeros cambios cardiovasculares que ocurren en el HPTP; 2) es un método rápidamente sensible al cambio después de una intervención (como la paratiroidectomía); y 3) es una técnica no invasiva y fácil de medir.

Los datos sobre la función endotelial en el HPTP son controversiales; por ejemplo, en el estudio realizado por Baykan, et al. ${ }^{27}$, quienes incluyeron 21 pacientes con HPTP y 27 controles sanos, en la población con HPTP se encontró una menor VMF, y esto también fue observado por Nilsson, et al. ${ }^{28}$, mientras que Neunteufl, et al. ${ }^{19}$ no encontraron un porcentaje significativo de disminución de la VMF en este tipo de pacientes. Sin embargo, en este estudio no se excluyó a los pacientes con otros factores de riesgo cardiovascular y disfunción endotelial (tabaquismo actual, hipertensión arterial o diabetes mellitus).

En cuanto a los estudios que han evaluado el cambio posterior a una intervención como la paratiroidectomía, Kosch, et al. ${ }^{29}$, en un estudio de casos y controles antes y 6 meses después de la paratiroidectomía, compararon 19 pacientes con HPTP sin factores de riesgo cardiovascular (como diabetes, hipertensión o enfermedad vascular conocida) con 20 controles sanos, y encontraron que antes de la cirugía el $18.2 \%$ de los pacientes con HPTP tenían disfunción endotelial frente al $4.7 \%$ de las personas sanas, con mejoría en el $16.7 \%$ de los casos posterior a la cirugía. Por su parte, Carrelli, et al. ${ }^{15}$ evaluaron la disfunción endotelial mediante VMF en 46 pacientes con HPTP leve, antes y a los 6 y 12 meses de la paratiroidectomía, sin encontrar diferencias significativas $(4.63 \pm 0.51 \%$ inicial vs. $4.38 \pm 0.83 \%$ a los 6 meses y $5.07 \pm 0.74 \%$ a los 12 meses; $p=0.72$ y 0.49 , respectivamente). No obstante, en un subanálisis de 15 pacientes en quienes se definió disfunción endotelial como vasodilatación < $2.2 \%$, se observó un incremento del $350 \%$ a los 6 y 12 meses después de la paratiroidectomía $(0.81$ $\pm 0.19 \%$ inicial y $3.18 \pm 0.79 \%$ a 6 meses $[p=0.02$ vs . inicial], y $3.68 \pm 1.22 \%$ a 12 meses [p $=0.04$ vs. inicial]). Los autores de este artículo concluyen que, en pacientes con HPTP leve, la VMF generalmente es normal y permanece sin cambios al año de la paratiroidectomía; solo se observa una normalización en aquellos pacientes con alteraciones en la vasodilatación iniciales. Por otra parte, Tuna, et al. ${ }^{22}$ evaluaron la VMF y el grosor íntima-media carotídeo en 53 pacientes con HPTP leve antes y después de la 
paratiroidectomía, y los compararon con 46 controles sanos, excluyendo a los pacientes con diabetes mellitus, enfermedad coronaria, insuficiencia renal, alteraciones tiroideas y antecedente de tabaquismo. En este estudio, la VMF fue menor en el grupo con HPTP $(2.6$ $\pm 1.2 \%$ vs. $14.8 \pm 9.6 \% ; p<0.001)$, mientras que el grosor íntima-media carotídeo era mayor en comparación con los controles $(0.69 \pm 0.18$ vs. $0.61 \pm 0.12 ; p$ $=0.045$ ). Cuando se realizó un subanálisis de 18 pacientes que no fueron sometidos a cirugía, no se observaron cambios en estos parámetros.

En nuestro estudio, que incluyó 15 pacientes con HPTP y criterios quirúrgicos para paratiroidectomía (el $60 \%$ con calcemias mayores de $11.2 \mathrm{mg} / \mathrm{dl}$ y solo un paciente sin daño de órgano diana), se observó una mejoría significativa en la disfunción endotelial evaluada mediante VMF, ya que hubo un aumento de los diámetros de la arteria braquial preisquemia y post-isquemia de 41 a $46 \mathrm{~mm}$ después de 6 meses de haberse llevado a cabo la paratiroidectomía. Sin embargo, a pesar de dicha mejoría, la mayor proporción de pacientes con disfunción endotelial no reunieron el criterio de vasodilatación $>10 \%$ posterior a la isquemia. Así mismo, solo hubo una tendencia a la mejoría en la presencia de disfunción diastólica evaluada mediante ecocardiograma Doppler pulsado. Es importante comentar que no se encontraron diferencias en los parámetros metabólicos de estos pacientes, por lo cual pudo asumirse que estos no influyeron en las diferencias encontradas en la vasodilatación y la función diastólica.

La controversia en el HPTP para la paratiroidectomía existe sobre todo en pacientes con HPTP leve (calcemia < $11.2 \mathrm{mg} / \mathrm{dl}$ ) y sin daño de órgano diana, que pudieran beneficiarse de una cirugía por presentar alteraciones cardiovasculares. En nuestra población, al tratarse de un centro de referencia de tercer nivel, la mayoría de los pacientes que llegan tienen HPTP más grave, con calcemias mayores de $11.2 \mathrm{mg} / \mathrm{dl}$ y además con osteoporosis o litiasis. Otra limitación es que, debido al tamaño de la muestra, no pudo hacerse un análisis estratificado por gravedad del hiperparatiroidismo. Además, consideramos que en nuestro estudio el tiempo de evaluación después de la cirugía, de 6 meses, fue corto en comparación con otros estudios en los que se ha evaluado desde 12 hasta 36 meses tras la paratiroidectomía. Sin embargo, debido a las características de nuestro hospital (alta saturación y necesidad de egreso a otras entidades federativas), el seguimiento durante más tiempo es complicado. Por lo anterior, sugerimos que se realicen más estudios con un mayor tamaño de muestra y un diseño que permita evaluar con precisión el impacto del tratamiento quirúrgico en los pacientes con enfermedad leve, como podría ser un ensayo clínico controlado aleatorizado, sobre todo para considerar el tratamiento quirúrgico en este tipo de pacientes. Finalmente, sugerimos realizar estudios utilizando técnicas que permitan una evaluación más precisa de la función cardiaca diastólica, como el Doppler tisular; sin embargo, debe considerarse que para su realización se requiere un entrenamiento previo, que los equipos necesarios no se encuentran en todas las unidades de primer contacto en nuestro instituto y que aún se requiere su estandarización ${ }^{30}$.

Consideramos que una de las fortalezas del estudio es su diseño, ya que al tratarse de un estudio cuasiexperimental, los pacientes funcionaron como sus propios controles, antes y después de la paratiroidectomía, lo cual disminuye la variabilidad biológica. Además, observamos que no hubo diferencias en cuanto a síndrome metabólico (o sus componentes) entre antes y después de la paratiroidectomía, por lo cual la mejoría puede atribuirse únicamente al retiro del estímulo de la hormona paratiroidea.

\section{Conclusiones}

Nuestro estudio indica que existe una mejoría en la función endotelial evaluada por VMF en los pacientes con HPTP a los 6 meses de la paratiroidectomía. Los estudios ecocardiográfico y de VMF pueden ser de utilidad en la evaluación preoperatoria de los pacientes con HPTP, y pudieran considerarse como estudios adicionales en los pacientes con HPTP leve.

\section{Conflicto de intereses}

Los autores declaran que no existen conflicto de intereses.

\section{Financiamiento}

No se contó con fuentes de financiamiento para la realización del estudio.

\section{Responsabilidades éticas}

Protección de personas y animales. Los autores declaran que los procedimientos seguidos se conformaron a las normas éticas del comité de experimentación 
humana responsable y de acuerdo con la Asociación Médica Mundial y la Declaración de Helsinki.

Confidencialidad de los datos. Los autores declaran que han seguido los protocolos de su centro de trabajo sobre la publicación de datos de pacientes.

Derecho a la privacidad y consentimiento informado. Los autores han obtenido el consentimiento informado de los pacientes y/o sujetos referidos en el artículo. Este documento obra en poder del autor de correspondencia.

\section{Bibliografía}

1. Melton LJ, $3^{\text {rd }}$. The epidemiology of primary hyperparathyroidism in North America. J Bone Miner Res. 2002;17(Suppl 2):N12-17.

2. Adami S, Marcocci C, Gatti D. Epidemiology of primary hyperparathyroidism in Europe. J Bone Miner Res. 2002;17(Suppl 2):N18-23.

3. Wermers RA, Khosla S, Atkinson EJ, Achenbach SJ, Oberg AL Grant CS, et al. Incidence of primary hyperparathyroidism in Rochester Minnesota, 1993-2001: an update on the changing epidemiology of the disease. J Bone Miner Res. 2006;21:171-7.

4. Hedback GM, Oden AS. Cardiovascular disease, hypertension and renal function in primary hyperparathyroidism. J Intern Med. 2002;251: 476-83.

5. Nilsson IL, Yin L, Lundgren E, Rastad J, Ekbom A. Clinical presentation of primary hyperparathyroidism in Europe - nationwide cohort analysis on mortality from nonmalignant causes. J Bone Miner Res. 2002;17(Suppl 2):N68-74.

6. Yu N, Donnan PT, Flynn RW, Murphy MJ, Smith D, Rudman A, et al. Increased mortality and morbidity in mild primary hyperparathyroid patients. The Parathyroid Epidemiology and Audit Research Study (PEARS). Clin Endocrinol (Oxf). 2010;73:30-4.

7. Farahnak $P$, Larfars G, Sten-Linder M, Nilsson IL. Mild primary hyperparathyroidism: vitamin $D$ deficiency and cardiovascular risk markers. J Clin Endocrinol Metab. 2011:96:2112-8.

8. Lundgren E, Ljunghall S, Akerstrom G, Hetta J, Mallmin H, Rastad J. Case-control study on symptoms and signs of "asymptomatic" primary hyperparathyroidism. Surgery. 1998;124:980-5.

9. Walker MD, Fleischer JB, Di Tullio MR, Homma S, Rundek T, Stein EM, et al. Cardiac structure and diastolic function in mild primary hyperparathyroidism. J Clin Endocrinol Metab. 2010;95:2172-9.

10. Mendoza-Zubieta V, González-Villasenor GA, Vargas-Ortega G, González B, Ramírez-Remtería C, Mercado M, et al. High prevalence of metabolic syndrome in a mestizo group of adult patients with primary hyperparathyroidism (PHPT). BMC Endocr Disord. 2015;15:16.

11. Luigi $P$, Chiara FM, Laura Z, Cristiano M, Giuseppina $C$, Luciano $C$, et al. Arterial hypertension, metabolic syndrome and subclinical cardiovascular organ damage in patients with asymptomatic primary hyperparathyroidism before and after parathyroidectomy: preliminary results. Int J Endocrinol. 2012;2012:408295.

12. Procopio M, Barale M, Bertaina S, Sigrist S, Mazzetti R, Loiacono M, et al. Cardiovascular risk and metabolic syndrome in primary hyperparathyroidism and their correlation to different clinical forms. Endocrine. 2014;47:581-9.

13. Bilezikian JP, Brandi ML, Eastell R, Silverberg SJ, Udelsman R, Marcocci $\mathrm{C}$, et al. Guidelines for the management of asymptomatic pri- mary hyperparathyroidism: summary statement from the fourth international workshop. J Clin Endocrinol Metab. 2014;99:3561-9.

14. Walker MD, Rundek T, Homma S, Di Tullio M, Iwata S, Lee JA, et al. Effect of parathyroidectomy on subclinical cardiovascular disease in mild primary hyperparathyroidism. Eur J Endocrinol. 2012;167:277-85.

15. Carrelli AL, Walker MD, Di Tullio MR, Homma S, Zhang C, McMahon DJ, et al. Endothelial function in mild primary hyperparathyroidism. Clin Endocrinol (Oxf). 2013;78:204-9.

16. Piovesan A, Molineri N, Casasso F, Emmolo I, Ugliengo G, Cesario F, et al. Left ventricular hypertrophy in primary hyperparathyroidism. Effects of successful parathyroidectomy. Clin Endocrinol (Oxf). 1999:50:321-8.

17. Stefenelli T, Abela C, Frank H, Koller-Strametz J, Globits S, Bergler-Klein $\mathrm{J}$, et al. Cardiac abnormalities in patients with primary hyperparathyroidism: implications for follow-up. J Clin Endocrinol Metab. 1997:82:106-12.

18. Agarwal G, Nanda G, Kapoor A, Singh KR, Chand G, Mishra A, et al. Cardiovascular dysfunction in symptomatic primary hyperparathyroidism and its reversal after curative parathyroidectomy: results of a prospective case control study. Surgery. 2013;154:1394-03.

19. Neunteufl T, Katzenschlager R, Abela C, Kostner K, Niederle B, Weidinger $F$, et al. Impairment of endothelium-independent vasodilation in patients with hypercalcemia. Cardiovasc Res. 1998:40:396-401.

20. Alberti KG, Eckel RH, Grundy SM, Zimmet PZ, Cleeman JI, Donato KA et al. Harmonizing the metabolic syndrome: a joint interim statement of the International Diabetes Federation Task Force on Epidemiology and Prevention; National Heart, Lung, and Blood Institute; American Heart Association; World Heart Federation; International Atherosclerosis Society; and International Association for the Study of Obesity. Circulation. 2009; $120: 1640-5$.

21. Friedewald WT, Levy RI, Fredrickson DS. Estimation of the concentration of low-density lipoprotein cholesterol in plasma, without use of the preparative ultracentrifuge. Clin Chem. 1972;18:499-502.

22. Tuna MM, Dogan BA, Arduc A, Imga NN, Tütüncü $Y$, Berker D, et al. Impaired endothelial function in patients with mild primary hyperparathyroidism improves after parathyroidectomy. Clin Endocrinol (Oxf). 2015;83:951-6.

23. Ferreira-Hermosillo A, Ramírez-Rentería C, Mendoza-Zubieta V, Molina-Ayala MA. Utility of the waist-to-height ratio, waist circumference and body mass index in the screening of metabolic syndrome in adult patients with type 1 diabetes mellitus. Diabetol Metab Syndr. 2014;6:32.

24. Lang RM, Badano LP, Mor-Avi V, Afilalo J, Armstrong A, Emande L, et al. Recommendations for cardiac chamber quantification by echocardiography in adults: an update from the American Society of Echocardiography and the European Association of Cardiovascular Imaging. J Am Soc Echocardiogr. 2015;28:1-39.

25. Rashid G, Bernheim J, Green J, Benchetrit S. Parathyroid hormone stimulates endothelial expression of atherosclerotic parameters through protein kinase pathways. Am J Physiol Renal Physiol. 2007;292: F1215-8.

26. Saleh FN, Schirmer H, Sundsfjord J, Jorde R. Parathyroid hormone and left ventricular hypertrophy. Eur Heart J. 2003;24:2054-60.

27. Baykan M, Erem C, Erdogan T, Hachihasanoglu A, Gedikli O, Kris A, et al. Impairment of flow mediated vasodilatation of brachial artery in patients with primary hyperparathyroidism. Int J Cardiovasc Imaging. 2007;23:323-8.

28. Nilsson IL, Aberg J, Rastad J, Lind L. Endothelial vasodilatory dysfunction in primary hyperparathyroidism is reversed after parathyroidectomy. Surgery. 1999;126:1049-55.

29. Kosch M, Hausberg M, Vormbrock K, Kisters K, Gabriels G, Rahn KH, et al. Impaired flow-mediated vasodilation of the brachial artery in patients with primary hyperparathyroidism improves after parathyroidectomy. Cardiovasc Res. 2000;47:813-8.

30. Kadappu KK, Thomas L. Tissue Doppler imaging in echocardiography: value and limitations. Heart Lung Cir. 2015;24:224-33. 